\title{
Neutron spectra measurement and calculations using data libraries CIELO, JEFF-3.2 and ENDF/B-VII.1 in iron benchmark assemblies
}

\author{
Bohumil Jansky ${ }^{1, \text { a }}$, Jiri Rejchrt ${ }^{1}$, Evzen Novak $^{1}$, Evzen Losa $^{1}$, Anatoly I. Blokhin ${ }^{2}$, and Elena Mitenkova ${ }^{2}$ \\ 1 Research Centre Rez, Department of Neutron Physics Sciences, Czech Republic \\ 2 Nuclear Safety Institute of Russian Academy of Sciences, Moscow, Russia
}

\begin{abstract}
The leakage neutron spectra measurements have been done on benchmark spherical assemblies iron spheres with diameter of $20,30,50$ and $100 \mathrm{~cm}$. The Cf- 252 neutron source was placed into the centre of iron sphere. The proton recoil method was used for neutron spectra measurement using spherical hydrogen proportional counters with diameter of $4 \mathrm{~cm}$ and with pressure of 400 and $1000 \mathrm{kPa}$. The neutron energy range of spectrometer is from 0.1 to $1.3 \mathrm{MeV}$. This energy interval represents about $85 \%$ of all leakage neutrons from $\mathrm{Fe}$ sphere of diameter $50 \mathrm{~cm}$ and about of $74 \%$ for $\mathrm{Fe}$ sphere of diameter $100 \mathrm{~cm}$. The adequate MCNP neutron spectra calculations based on data libraries CIELO, JEFF-3.2 and ENDF/B-VII.1 were done. Two calculations were done with CIELO library. The first one used data for all Fe-isotopes from CIELO and the second one (CIELO-56) used only Fe-56 data from CIELO and data for other Fe isotopes were from ENDF/B-VII.1. The energy structure used for calculations and measurements was 40 gpd (groups per decade) and 200 gpd. Structure 200 gpd represents lethargy step about of $1 \%$. This relatively fine energy structure enables to analyze the Fe resonance neutron energy structure. The evaluated cross section data of Fe were validated on comparisons between the calculated and experimental spectra.
\end{abstract}

\section{Introduction}

Neutron and gamma spectra behind iron and water layers are long term measured on mock-up of WWER-1000 reactor at LR-0 research reactor in Research Centre, Rez (Czech Republic). Neutron and gamma fields parameters were studied behind iron (reactor pressure vessel model) and water layers. Also corresponding measurements have been done on benchmark iron spherical assemblies with diameter of $20,30,50$ and $100 \mathrm{~cm}$. The Cf- 252 neutron source was placed into the centre of spheres. The measurement results were always compared with parallel MCNP calculations using different data libraries. Two calculations were done with CIELO library [1]. The first one used data for all $\mathrm{Fe}$ isotopes from CIELO and the second one CIELO-56 (laboratory name) used only Fe56 data from CIELO. Data for other $\mathrm{Fe}$ isotopes were from ENDF/B-VII.1 [2]. The files used for material description in MCNP were in the CIELO: 26054.77C, 26056.77C, 26057.77C, 26058.77C taken from CIELO and in the CIELO56: 26056.77C from CIELO, 26054.72C, 26057.72C, 26058.72C from ENDF/B.

\section{Experimental assemblies}

Experimental assemblies are formed by the pure iron sphere with diameter of $20,30,50$, and $100 \mathrm{~cm}$, see Table 1, with neutron source double encapsulated in

a e-mail: Bohumil.Jansky@cvrez.cz stainless steel capsule (th. $2 \times 0.8 \mathrm{~mm}$ ) placed in centre (transferred by Flexo Rabbit system), see the Fig. 1. Several neutron sources with different emission $\mathrm{Q}=$ 1E71E9 $\mathrm{n} / \mathrm{s}$ were used. The sphere centre and detector centre are always placed at the height of $200 \mathrm{~cm}$ above the concrete floor. Shadow (shielding) cone was used for background measurement and subtracting.

Two hydrogen proportional spherical detectors (HPD) $\mathrm{K} 4$ and $\mathrm{K} 8$ with diameter of $40 \mathrm{~mm}$ were used in neutron spectrometer [3]. The detector K4 with pressure $400 \mathrm{kPa}$ was used for measurement in the energy range $\mathrm{En}=$ $0.1-0.4 \mathrm{MeV}$, the detector $\mathrm{K} 8$ with pressure $1000 \mathrm{kPa}$ was used for measurement in the energy range En $=0.4$ $1.3 \mathrm{MeV}[3]$.

\section{Methodology of measurement and calculation}

To get effect the background measured with shielding cone is subtracted of whole measurement. The calculations were performed using Monte-Carlo program MCNP. For input neutron spectrum is used description from Sajo [4].

As for geometry description, a simplified model was used, which substitutes assembly elements with concentric spherical shells around the source. Also, the MCNP detector is represented by a $1 \mathrm{~cm}$ thick spherical shell with radius equal to the real detector-source distance $\mathrm{R}$. For each calculation $10^{8}-10^{9}$ particle histories were computed.

(C) The Authors, published by EDP Sciences. This is an Open Access article distributed under the terms of the Creative Commons Attribution License 4.0 (http://creativecommons.org/licenses/by/4.0/). 
Table 1. Iron (Fe) Benchmark assemblies used for measurements and calculations.

\begin{tabular}{|l|c|c|c|}
\hline Acronym & $\begin{array}{c}\text { Fe sphere } \\
\text { diam. } \\
(\mathrm{cm})\end{array}$ & $\begin{array}{c}\text { Detector to sphere } \\
\text { distance } \\
\text { centre to centre }(\mathrm{cm})\end{array}$ & $\begin{array}{c}\text { Max. } \\
\text { impurities } \\
* *)\end{array}$ \\
\hline FE20
\end{tabular}

${ }^{*)}$ Measurement on the sphere surface ${ }^{* *)}$ mainly Mn.

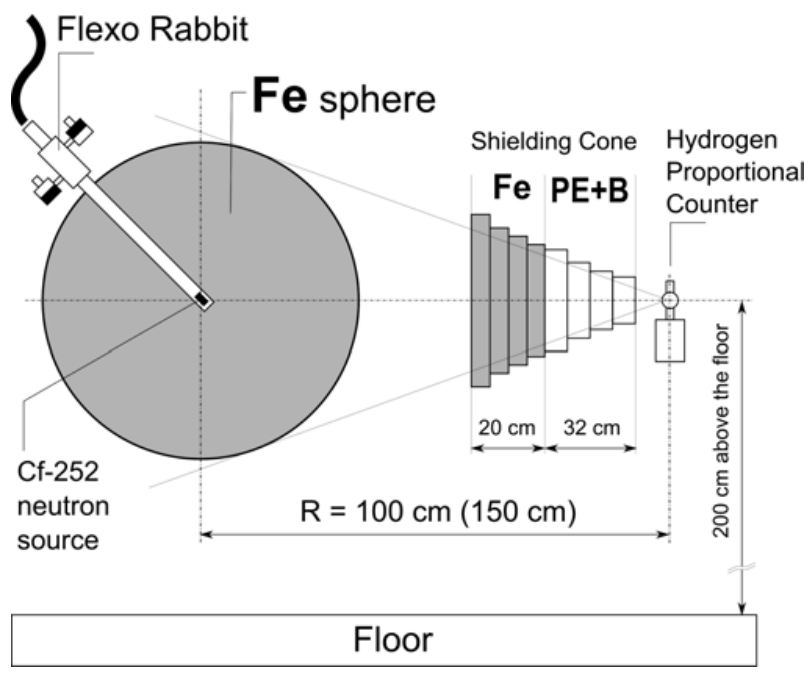

Figure 1. Basic scheme of n-leakage spectrum measurement.

\section{Normalisation and smoothing of calculated results, energy structure}

The result of neutron spectra calculation and measurement $\varphi(\mathrm{E})$ is normalised in the following way

$$
4 \pi R^{2} \varphi(E) / Q[1 / \mathrm{MeV}]
$$

where $\mathrm{R}$ is distance between detector and neutron source (centre to centre) and Q [1/s] is neutron source emission rate. Quantity depicted in the figures has the following form and dimension

$$
E 4 \pi R^{2} \varphi(E) / Q[1]
$$

The integral values presented in following tables are also with dimension of 1 .

The measured and calculated spectra were evaluated in two group structures: $40 \mathrm{gpd}$ (groups per decade), it corresponds to the lethargy step about $6 \%$ and in structure $200 \mathrm{gpd}$, i.e., with lethargy step about $1 \%$. Structure 200 gpd is proper only for measurements with very good statistics, but it represents long exposure time. The calculated spectra were usually smoothed by Gaussian with constant percentage resolution $\Delta$ of FWHM: $\Delta=$ $13 \%$ for $40 \mathrm{gpd}$ and $\Delta=4 \%$ for $200 \mathrm{gpd}$. The aim of this smoothing is to obtain the form of calculated spectrum similar to measured spectrum with detector of given resolution.
Table 2. Assembly FE100R150, integral values [1], 40 gpd.

\begin{tabular}{|cc|l|lllll|}
\hline \multicolumn{2}{|c|}{$\begin{array}{c}\text { Energy range } \\
{[\mathrm{MeV}]}\end{array}$} & & \multicolumn{4}{|c|}{ Nuclear data libraries } \\
\hline from & to & EXP & CIELO & CIELO-56 & ENDF & JEFF \\
\hline 0.1 & 1.3 & 0.5950 & 0.6795 & 0.7081 & 0.6670 & 0.6628 \\
0.1 & 0.2 & 0.1848 & 0.2158 & 0.1872 & 0.1715 & 0.2001 \\
0.2 & 0.4 & 0.2498 & 0.3132 & 0.3444 & 0.3130 & 0.2864 \\
0.4 & 0.8 & 0.1363 & 0.1293 & 0.1548 & 0.1594 & 0.1524 \\
0.8 & 1 & 0.0154 & 0.0162 & 0.0155 & 0.0146 & 0.0159 \\
1 & 1.3 & 0.0087 & 0.0050 & 0.0062 & 0.0086 & 0.0080 \\
\hline
\end{tabular}

Table 3. Assembly FE100R150, C/E comparison, 40 gpd.

\begin{tabular}{|cc|l|llll|}
\hline \multicolumn{2}{|c|}{$\begin{array}{c}\text { Energy range } \\
{[\mathrm{MeV}]}\end{array}$} & & \multicolumn{4}{|c|}{ Nuclear data libraries } \\
\hline from & to & EXP & CIELO & CIELO-56 & ENDF JEFF \\
\hline 0.1 & 1.3 & 1.000 & 1.142 & 1.190 & 1.121 & 1.114 \\
0.1 & 0.2 & 1.000 & 1.168 & 1.013 & 0.928 & 1.083 \\
0.2 & 0.4 & 1.000 & 1.254 & 1.379 & 1.253 & 1.147 \\
0.4 & 0.8 & 1.000 & 0.949 & 1.136 & 1.169 & 1.118 \\
0.8 & 1 & 1.000 & 1.053 & 1.006 & 0.948 & 1.032 \\
1 & 1.3 & 1.000 & 0.571 & 0.713 & 0.989 & 0.920 \\
\hline
\end{tabular}

Table 4. Fe assemblies - MCNP calculation with CIELO library, integral values [1], 40 gpd.

\begin{tabular}{|cc|llll|}
\hline \multicolumn{2}{|c|}{$\begin{array}{c}\text { Energy range } \\
{[\mathrm{MeV}]}\end{array}$} & \multicolumn{4}{|c|}{ Fe assemblies } \\
\hline from & to & FE20 & FE30 & FE50 & FE100 \\
\hline 0.1 & 1.3 & 0.6568 & 0.7603 & 0.8442 & 0.6795 \\
0.1 & 0.2 & 0.0602 & 0.0874 & 0.1411 & 0.2158 \\
0.2 & 0.4 & 0.1525 & 0.2102 & 0.3037 & 0.3132 \\
0.4 & 0.8 & 0.2481 & 0.2849 & 0.2852 & 0.1293 \\
0.8 & 1 & 0.0928 & 0.0916 & 0.0681 & 0.0162 \\
1 & 1.3 & 0.1032 & 0.0862 & 0.0462 & 0.0050 \\
\hline
\end{tabular}

\section{Uncertainties}

Uncertainty of single measurement is composed of uncertainty of the "A-type" that include statistical uncertainty of single measurement and consequent calculation of each energy group and uncertainty of "B-type" that include influence of instability in benchmark geometry and detector position, n-source orientation, setting of electronics, detector discharges, energy calibration during time remote repeated measurements. Integral values are presented in Tables 2 and 5 with combined uncertainty in interval 3-7\%, where the statistical uncertainty of "A-type" is about $1-3 \%$ (for $40 \mathrm{gpd}$ and large energy interval) and the uncertainty of "Btype" is about 3-6\%. The statistical uncertainty for 200 gpd (Table 7) is $1-6 \%$. Each measurement was repeated 2-9 times. Uncertainties of MCNP calculations for each group are better than $1 \%$ in energy interval $0.1-1.3 \mathrm{MeV}$.

\section{Results}

Results are presented at Tables $2-8$ and Figs. $2-5$. The proper norms (dividing coefficients): 1, 3, 10 and 30 are used in Fig. 2 and Fig. 4 for spectra FE20, FE30, FE50 and FE100 for the better clarity of graphs. More results were presented in $40 \mathrm{gpd}$ structure in [5]. 
Table 5. Fe assemblies - Experimental values (HPD spectrometer), integral values [1], 40 gpd.

\begin{tabular}{|cc|llll|}
\hline \multicolumn{2}{|c|}{$\begin{array}{c}\text { Energy range } \\
{[\mathrm{MeV}]}\end{array}$} & \multicolumn{4}{|c|}{ Fe assemblies } \\
\hline from & to & FE20 & FE30 & FE50 & FE100 \\
\hline 0.1 & 1.3 & 0.5850 & 0.6843 & 0.7679 & 0.5950 \\
\hline 0.1 & 0.2 & 0.0559 & 0.0790 & 0.1265 & 0.1848 \\
0.2 & 0.4 & 0.1269 & 0.1723 & 0.2489 & 0.2498 \\
0.4 & 0.8 & 0.2148 & 0.2587 & 0.2746 & 0.1363 \\
0.8 & 1 & 0.0820 & 0.0806 & 0.0609 & 0.0154 \\
1 & 1.3 & 0.1054 & 0.0937 & 0.0570 & 0.0087 \\
\hline
\end{tabular}

Table 6. Fe assemblies - C/E comparison of integral values (Tables 4 and 5), MCNP calculation with CIELO.

\begin{tabular}{|cc|l|llll|}
\hline \multicolumn{2}{|c|}{$\begin{array}{c}\text { Energy range } \\
{[\mathrm{MeV}]}\end{array}$} & & \multicolumn{4}{c|}{ Fe assemblies } \\
\hline from & to & EXP & FE20 & FE30 & FE50 & FE100 \\
\hline 0.1 & 1.3 & 1.000 & 1.123 & 1.111 & 1.099 & 1.142 \\
0.1 & 0.2 & 1.000 & 1.077 & 1.107 & 1.115 & 1.168 \\
0.2 & 0.4 & 1.000 & 1.202 & 1.220 & 1.220 & 1.254 \\
0.4 & 0.8 & 1.000 & 1.155 & 1.101 & 1.039 & 0.948 \\
0.8 & 1 & 1.000 & 1.132 & 1.135 & 1.120 & 1.054 \\
1 & 1.3 & 1.000 & 0.979 & 0.920 & 0.810 & 0.572 \\
\hline
\end{tabular}

Table 7. FE100R53 assembly, fine structure 200 gpd.

\begin{tabular}{|cc|l|lll|}
\hline \multicolumn{2}{|c|}{$\begin{array}{c}\text { Energy range } \\
{[M e V]}\end{array}$} & & \multicolumn{3}{c|}{ Integral values [1] } \\
\hline from & to & EXP & ENDF & CIELO & JEFF \\
\hline 0.013 & 1.290 & 1.0442 & 1.0914 & 1.1124 & 1.1131 \\
0.013 & 0.033 & 0.1547 & 0.1403 & 0.1445 & 0.1526 \\
0.033 & 0.060 & 0.0299 & 0.0265 & 0.0249 & 0.0292 \\
0.060 & 0.090 & 0.0813 & 0.0770 & 0.0776 & 0.0795 \\
0.090 & 0.150 & 0.1576 & 0.1318 & 0.1662 & 0.1589 \\
0.150 & 0.200 & 0.1047 & 0.0959 & 0.1189 & 0.1069 \\
0.200 & 0.250 & 0.0649 & 0.0663 & 0.0656 & 0.0663 \\
0.250 & 0.290 & 0.0794 & 0.0870 & 0.0835 & 0.0812 \\
0.290 & 0.330 & 0.0905 & 0.1351 & 0.1276 & 0.1165 \\
0.330 & 0.400 & 0.0816 & 0.1081 & 0.1160 & 0.1040 \\
0.400 & 0.520 & 0.0537 & 0.0570 & 0.0511 & 0.0595 \\
0.520 & 0.780 & 0.1146 & 0.1364 & 0.1100 & 0.1263 \\
0.780 & 1.060 & 0.0238 & 0.0231 & 0.0224 & 0.0256 \\
1.060 & 1.290 & 0.0074 & 0.0070 & 0.0042 & 0.0066 \\
\hline
\end{tabular}

Table 8. 1-C/E, FE100R53 assembly, fine structure 200 gpd.

\begin{tabular}{|lc|l|rrr|}
\hline \multicolumn{2}{|l|}{$\begin{array}{l}\text { Energy range } \\
{[\mathrm{MeV}]}\end{array}$} & & & \multicolumn{3}{|l|}{ 1-C/E [\%] } & \\
\hline from & to & EXP & ENDF & CIELO & JEFF \\
\hline 0.013 & 1.290 & 0.00 & 4.52 & 6.54 & 6.60 \\
0.013 & 0.033 & 0.00 & -9.30 & -6.61 & -1.33 \\
0.033 & 0.060 & 0.00 & -11.49 & -16.87 & -2.43 \\
0.060 & 0.090 & 0.00 & -5.36 & -4.58 & -2.19 \\
0.090 & 0.150 & 0.00 & -16.36 & 5.44 & 0.80 \\
0.150 & 0.200 & 0.00 & -8.37 & 13.54 & 2.12 \\
0.200 & 0.250 & 0.00 & 2.18 & 1.19 & 2.16 \\
0.250 & 0.290 & 0.00 & 9.61 & 5.23 & 2.32 \\
0.290 & 0.330 & 0.00 & 49.15 & 40.88 & 28.66 \\
0.330 & 0.400 & 0.00 & 32.41 & 42.08 & 27.37 \\
0.400 & 0.520 & 0.00 & 6.12 & -4.93 & 10.84 \\
0.520 & 0.780 & 0.00 & 19.02 & -4.00 & 10.18 \\
0.780 & 1.060 & 0.00 & -2.78 & -5.70 & 7.63 \\
1.060 & 1.290 & 0.00 & -6.34 & -42.95 & -10.88 \\
\hline
\end{tabular}

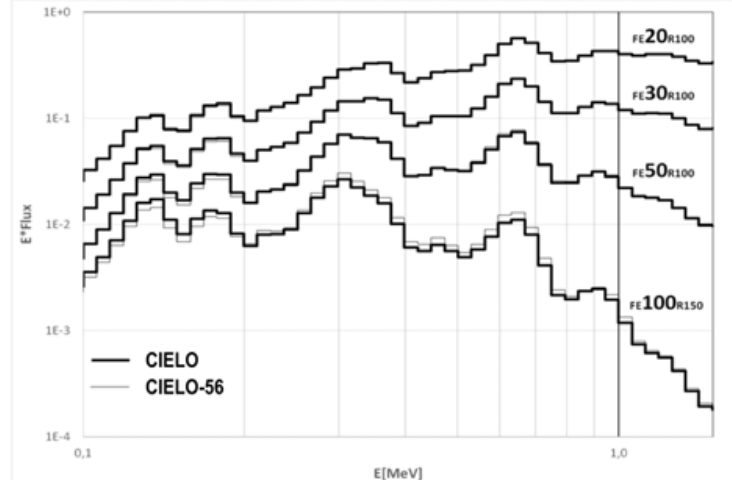

Figure 2. CIELO and CIELO-56: Comparison of calculated spectra Fe assemblies - diam. 20,30,50,100 cm, 40 gpd.

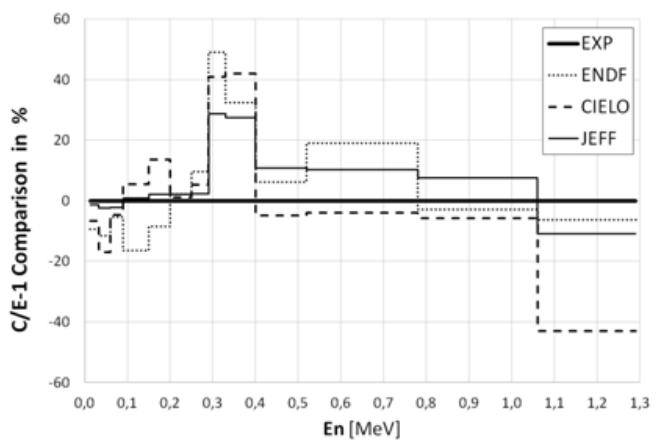

Figure 3. CALC/EXP-1, assembly FE100, $\mathrm{R}=53$, fine structure 200 gpd, see Table 8.

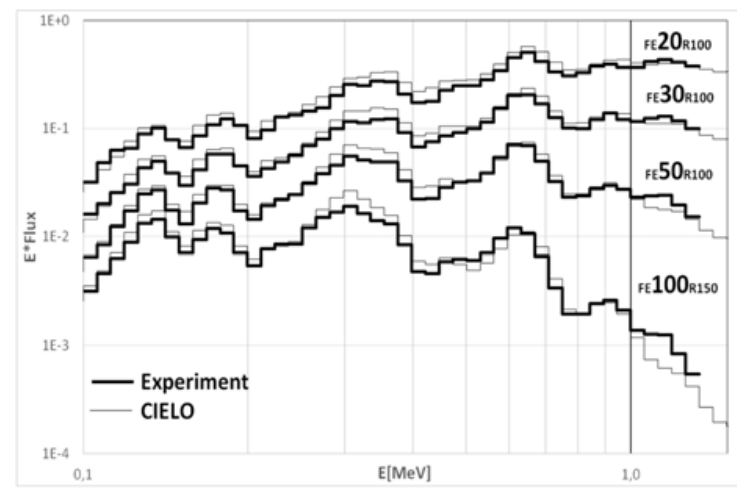

Figure 4. Comparison of calculated and measured spectra (CIELO and Experiment), Fe 20, 30, 50 and $100 \mathrm{~cm}, 40 \mathrm{gpd}$ (proper norms were used).

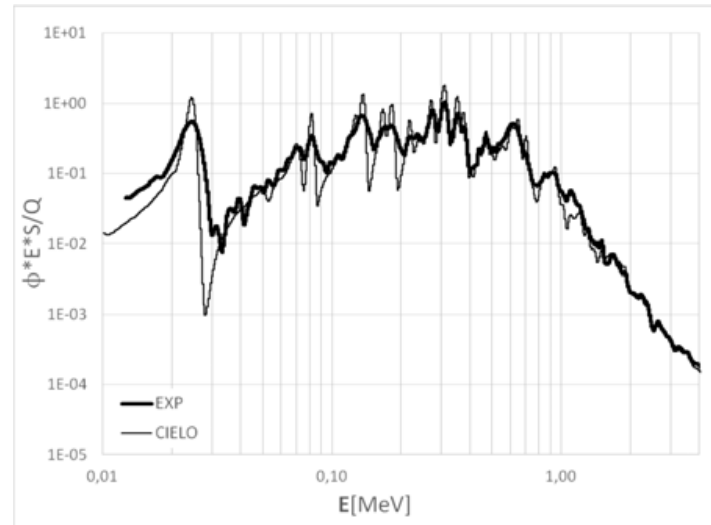

Figure 5. Assembly FE100, R = 53, Comparison of Measured (thick) and calculated with CIELO (thin) spectra in structure 200 gpd. 


\section{Conclusions}

Based on data presented in tables and pictures it is possible to conclude:

1) Table 3: For assembly FE100, R150 and all libraries in interval $0.1-1.3 \mathrm{MeV}$ is the $\mathrm{C} / \mathrm{E}=1.11-1.19$.

2) Table 6: For FE20, 30, 50, 100 and library CIELO in interval $0.1-1.3 \mathrm{MeV}$ is the $\mathrm{C} / \mathrm{E}=1.10-1.14$.

3) Items 1) and 2) indicate systematic calculation overestimation of 10-14\% in this region (of course, it could be measurement underestimation). The identical assembly FE50R100 was measured in past years at Rez by colleagues from Skoda Plzen (M.Holman) and from FEI Obninsk (L.Trykov) using independent (but also proton recoil) spectrometers. Their results are not more different than about $1-5 \%$ in region $0.1-1.3 \mathrm{MeV}$ in comparison with our results.

4) Tables 2 and 3 represent comparison of CIELO and CIELO-56 libraries. Regarding our experimental experiences with Iron filtered beams from past years, when using older versions of Data Libraries (ENDF, BROND, JENDL) two general rules were observed: $\mathbf{C} / \mathbf{E}<\mathbf{1}$ for $\mathbf{0 . 1 - 0 . 2 ~ M e V ~ a n d ~} \mathbf{C} / \mathbf{E}>\mathbf{1}$ for 0.2-0.4 and 0.4-0.8 MeV (two characteristic wide Iron peaks around $0.3 \mathrm{MeV}$ and $0.6 \mathrm{MeV}$ ). When we compare CIELO/CIELO-56, we can observe the "proper improving trend" in above mentioned regions when new $\mathrm{Fe}-54$ (and Fe-57 and $\mathrm{Fe}-58$, of course) new cross section (CS) in CIELO is used. It indicates the important role of $\mathbf{F e - 5 4} \mathbf{C S}$ for Iron neutron transport calculations although the content of $\mathrm{Fe}-54$ in natural $\mathrm{Fe}$ is only $5.8 \%$. Also, influence of other two isotopes in natuaral iron should be taken into account (the content of $\mathrm{Fe}-57$ is $2.2 \%$ and content of Fe-58 is $0.28 \%$ ).

5) When analysing the influence of Fe-54 in whole energy interval it seems that the significant differences between calculations using CIELO and CIELO-56 are observed for En $<1 \mathrm{MeV}$. Analyse of results indicates that the role of Fe-54 increases with Fe slab thickness, see Fig. 2. For $\mathrm{En}>1 \mathrm{MeV}$ is the influence of Fe-54 minimal.
6) Figure 4 indicates that peak on $0.6 \mathrm{MeV}$ is shifted (relating to experiment) for CIELO. The probably reason is the relation between heights of the 0.610 , 0.641 and $0.702 \mathrm{MeV}$ peaks that at are visible only in fine neutron energy structure $200 \mathrm{gpd}$, see Fig 5. The peak $0.702 \mathrm{MeV}$ is probably unrealistic dominant for CIELO.

7) Figure 4 and Tables 3 and 6 indicate that in energy interval $1-1.3 \mathrm{MeV}$ the ratio $\mathrm{C} / \mathrm{E}$ for CIELO (not for ENDF and JEFF) decreases rapidly with Fe thickness from the value 0.98 (Fe20) to the 0.57 (Fe100). Neutron absorption cross section is unrealistic high in the region $1-1.3 \mathrm{MeV}$.

We can conclude that CIELO brings not a great improvement of $\mathrm{C} / \mathrm{E}$ ratio in the region $0.1-1.3 \mathrm{MeV}$ in comparison with ENDF and JEFF. It is necessary to emphasize the great role of $\mathrm{Fe}-54$ cross section in natural $\mathrm{Fe}$.

The presented work was financially supported by the Ministry of Education, Youth and Sport Czech Republic Project LQ1603 (Research for SUSEN).

\section{References}

[1] https://www-nds.iaea.org/CIELO

[2] http://www.nndc.bnl.gov/endf/b7.1/ acefiles.html

[3] B. Jansky, E. Novak, Neutron Spectrometry with Spherical Hydrogen Proportional Detectors, Nuclear Instruments and Methods in Physics Research A735, 390-398 (2014)

[4] E. Sajo, M.L. Williams, M. Asgari, Comparison of measured and calculated neutron transmission through steel for a 252Cf source, Ann. Nucl. Energy 20(9), 285-604 (1993)

[5] B. Jansky et al., Neutron spectra measurement and calculations using data libraries CIELO, JEFF3.2 and ENDF/B-VII.1 in spherical Iron benchmark assemblies, ISINN-24, Dubna, Russia, May 23-27, 2016, will be published 